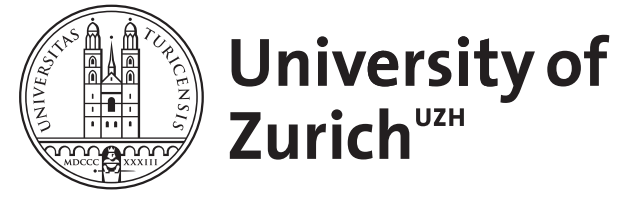

Zurich Open Repository and Archive

University of Zurich

University Library

Strickhofstrasse 39

CH-8057 Zurich

www.zora.uzh.ch

Year: 2012

\title{
Excimer Laser Trabeculostomy
}

Berlin, M S ; Töteberg-Harms, M ; Kleinberger, L ; Stodtmeister, R P ; Giers, U F

Posted at the Zurich Open Repository and Archive, University of Zurich

ZORA URL: https://doi.org/10.5167/uzh-66922

Journal Article

Originally published at:

Berlin, M S; Töteberg-Harms, M; Kleinberger, L; Stodtmeister, R P; Giers, U F (2012). Excimer Laser Trabeculostomy. Glaucoma Today:51-52. 


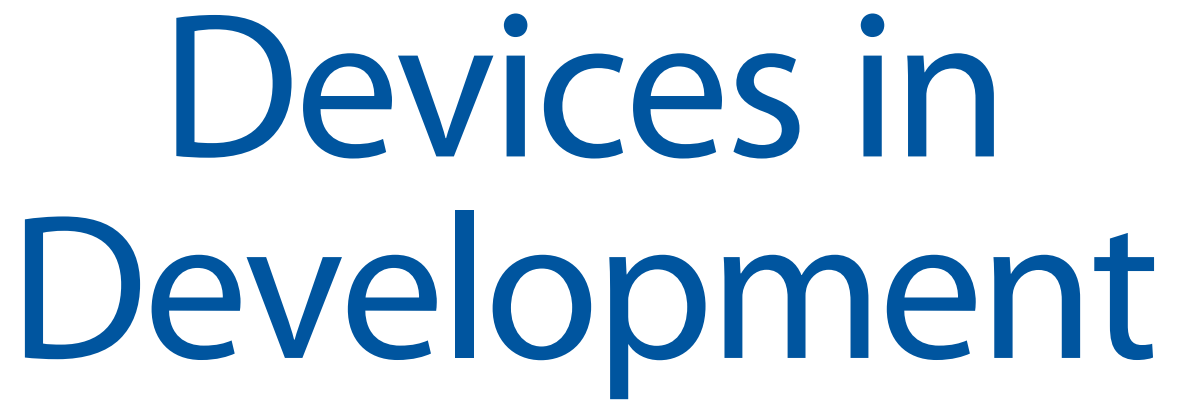

An update on some of the technologies and procedures being investigated

for microinvasive glaucoma surgery.

\section{AqueSys Implant}

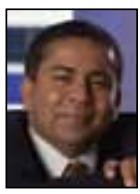

\section{By Rohit Varma, MD, MPH}

Many of the devices for microinvasive glaucoma surgery deliver on the promise of a less invasive procedure but, as of yet, without the desired efficacy. This is likely due to differences in the mechanism of action between gold standard procedures targeting subconjunctival outflow and the canal or suprachoroidal approaches.

AqueSys, Inc., has reinvented the approach to subconjunctival outflow. The company's technology is designed to provide a minimally invasive ab interno procedure while safely and significantly lowering the IOP of patients with early, moderate, and refractory glaucoma.

\section{DESIGN}

The AqueSys Implant is made of a collagen-derived gelatin. Upon implantation, the device is designed to create a diffuse outflow of aqueous from the anterior chamber into the nondissected tissue of the subconjunctival space. A well-known gelatin was chosen because it is extremely well tolerated by the human body and is noninflammatory. It is also very soft, and this pliability allows the device to conform to the ocular tissue, which should minimize many of the problems associated with synthetic materials (eg, migration, erosion, corneal endothelial damage). The gelatin material is cross-linked during manufacturing, which makes it permanent.

\section{PROCEDURE}

The AqueSys Implant (Figure 1) is placed through a small (approximately $1.6 \mathrm{~mm}$ ) clear corneal incision via a preloaded IOL-like inserter. Using an ab interno approach, the surgeon advances the inserter across the anterior chamber into the quadrant of the eye where implantation is planned. Upon placing the inserter's tip at the desired subconjunctival location, the surgeon delivers the device in a fashion similar to placing an $\mathrm{IOL}$ in the bag. The AqueSys procedure is straightforward and can be performed as a primary procedure or in conjunction with cataract surgery.

One clear benefit of the AqueSys ab interno to subconjunctival approach is that it bypasses all potential aqueous outflow obstructions instead of trying to reinvigorate an already failing outflow pathway, as occurs in other approaches. ${ }^{1,2}$ The conjunctiva-sparing ab interno approach means that additional AqueSys Implants could be placed over the course of the patient's lifetime, if

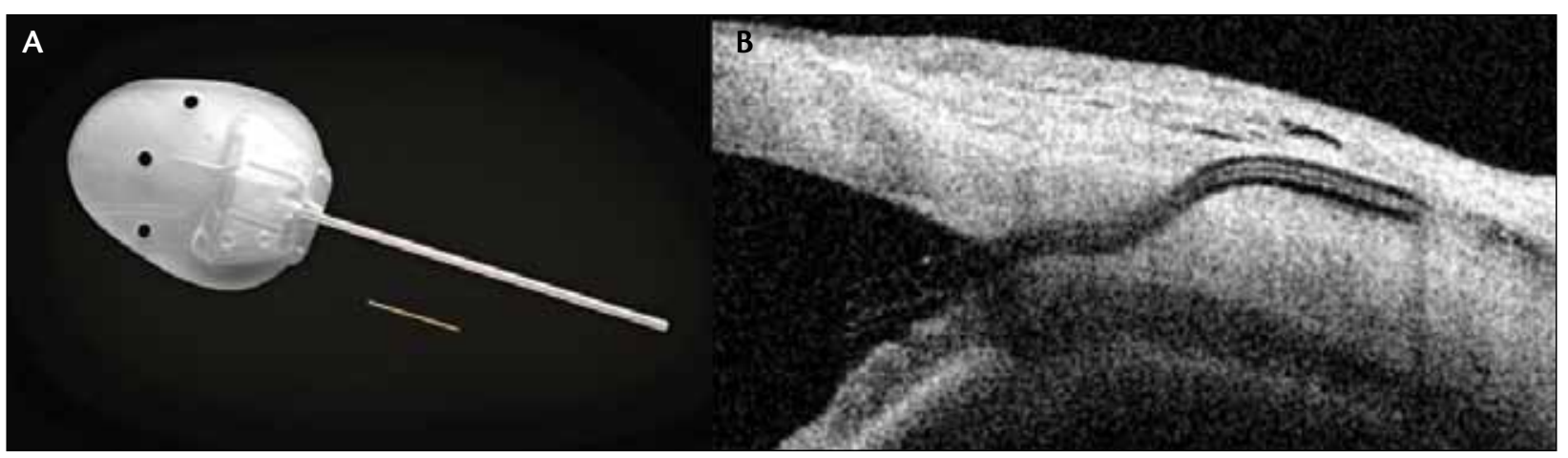

Figure 1. The 6-mm AqueSys Implant is positioned next to an Ahmed Glaucoma Valve (New World Medical, Inc.) (A). Anterior segment optical coherence tomography shows the soft AqueSys Implant conforming to the tissue in a human eye (B). 


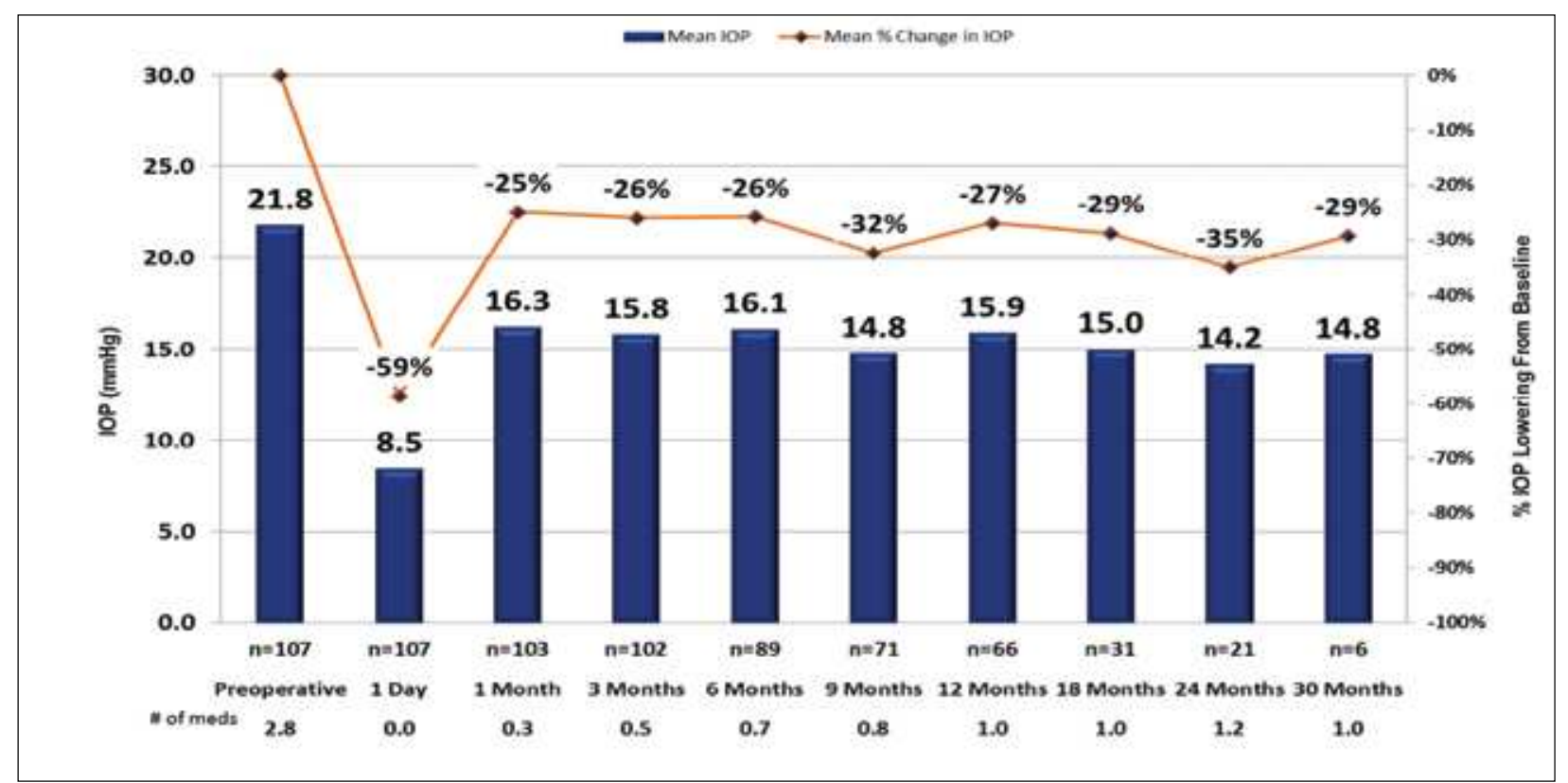

Figure 2. Mean IOP, mean percentage reduction from best medicated IOP, and mean number of IOP-lowering medications over time in a multicenter clinical study. Patients' IOP-lowering medications were not washed out prior to surgery.

needed. Furthermore, unlike with more invasive ab externo approaches or implants for microinvasive glaucoma surgery that require significant retention features (sutures, barbs, etc.), the AqueSys device can be removed in a straightforward procedure with minimal ocular trauma.

\section{RESULTS}

Several multicenter studies are underway in the United States, Canada, Europe, Asia, Australia, and South America. Figure 2 shows the initial 2-year follow-up results from a multicenter study outside the United States in patients with mild, moderate, and refractory glaucoma $(n=107)$. The data reflect significant IOP lowering from best medicated preoperative IOPs that was sustained over a 2-year period. Follow-up for this study is ongoing.,4

AqueSys was granted the CE Mark in Europe in 2011 for a broad treatment range for patients with mild, mod- erate, and refractory glaucoma. The company is currently enrolling subjects under an approved investigational device exemption for its first indication in the United States, with an estimated clearance in 2014.

Rohit Varma, MD, MPH, is a professor with the Department of Ophthalmology and Preventive Medicine at the University of Southern California, Keck School of Medicine, in Los Angeles. He is a paid consultant/advisor to AqueSys, Inc. Dr. Varma may be reached at (323) 4426411;rvarma@usc.edu.

1. Goel M, Picciani RG, Lee RK, Bhattacharya SK. Aqueous humor dynamics: a review. Open Ophthalmol f. 2010;4:52-59.

2. Gedde SJ, Schiffman JC, Feuer WJ, et al; Tube Versus Trabeculectomy Study Group. Treatment outcomes in the Tube Versus Trabeculectomy (TVT) Study after five years of follow-up. Am J Ophthalmol. 2012;153(5):789-803.e2. 3. Gomez IK, Dick B. First results of the innovative minimal-invasive glaucoma surgery technique: AqueSys Aquecentesis Procedure. Presented at: XXX Congress of the ESCRS; September 8-12, 2012; Milan, Italy.

4. Palmberg PF. One year results with the minimally invasive Aquecentesis procedure. Presented at: 6th International Congress on Glaucoma Surgery (ICGS) 2012; September 13-15, 2012; Glasgow, Scotland.

\section{CyPass Micro-Stent}

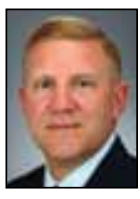

By William J. Flynn, MD

Designed for microinvasive glaucoma surgery, the CyPass Micro-Stent (Transcend Medical) has achieved promising early results in clinical trials. The device is implanted in the supraciliary space to establish a permanent conduit for aqueous filtration via uveoscleral outflow. The uveoscleral outflow pathway is an ideal target for therapeutic intervention for a number of reasons. Experimental evidence indicates that there is a negative pressure gradient between the suprachoroidal space and the anterior chamber, which provides a driving force for aqueous outflow. ${ }^{1}$ Clinical experience demonstrates that a traumatic or iatrogenic cyclodialysis is associated with a significant decrease in IOP, because it creates nontrabecular outflow through the uveoscleral route. ${ }^{2}$ Although uveoscleral outflow is relatively independent of IOP when 


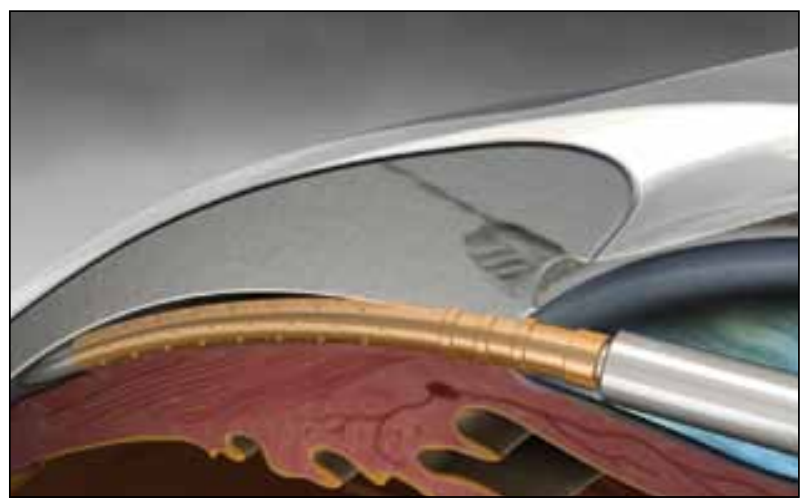

Figure 1. The CyPass Micro-Stent is loaded on the guidewire of the inserter and implanted in the supraciliary space.

pressure is at normal levels, a cyclodialysis results in a markedly pressure-dependent increase in uveoscleral outflow. ${ }^{3,4}$ Moreover, experience in glaucoma pharmacotherapy demonstrates that some of the most effective IOP-lowering topical therapies act primarily by increasing uveoscleral outflow. ${ }^{5}$

\section{DESIGN}

The CyPass (Figure 1) is a fenestrated, miniature stent made of biocompatible, nonbiodegradable polyimide material that has demonstrated a minimal inflammatory or fibrotic reaction in preclinical studies in a rabbit model. ${ }^{6}$ The device is $6.35 \mathrm{~mm}$ long and has an external diameter of $510 \mu \mathrm{m}$. The inserter for the stent consists of a handpiece and a releasable guidewire. The latter was designed to atraumatically follow the anatomical plane of the potential space between the ciliary body and the sclera.

\section{PROCEDURE}

The procedure is performed ab interno. After obtaining a view of the angle with a goniolens, the surgeon identifies the key anatomical landmark, the scleral spur. Loaded in the inserter, the CyPass is then advanced via a paracentesis or phacoemulsification incision across the anterior chamber to a position juxtaposed with the planned site of insertion. The goniolens is reintroduced to view both the CyPass and the scleral spur. The surgeon then implants the device by inserting the guidewire of the inserter just posterior to the scleral spur and advancing the stent so that nearly its entire length is in the supraciliary space (Figure 2). The implantation via the blunt, "noncutting" dissection of the guidewire creates a controlled cyclodialysis that is maintained by the CyPass.

\section{RESULTS}

Multiple clinical studies in Europe and the United States are underway or were recently completed. The reports on IOP reduction have been favorable and consistent.

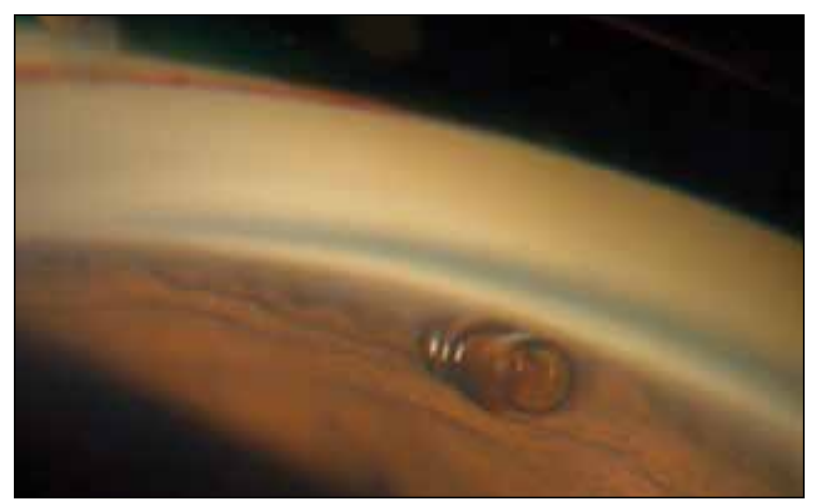

Figure 2. The surgeon views the device through a goniolens after implantation.

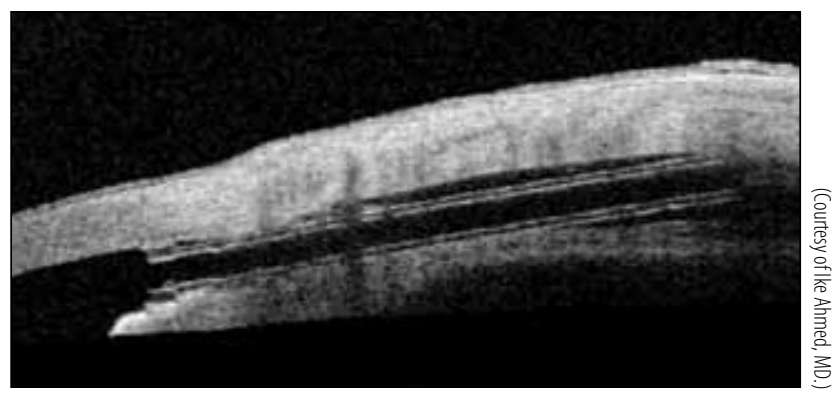

Figure 3. Optical coherence tomography image of a CyPass Micro-Stent in the supraciliary space.

lanchulev shared the first clinical results of more than 81 eyes, which achieved a reduction in IOP from 22.9 to $16.2 \mathrm{~mm} \mathrm{Hg}$ at 6 months. ${ }^{7}$ More recently, Hoeh et al reported that combining the implantation of the CyPass with phacoemulsification provided a sustained decrease in IOP that exceeded 35\% through 12 months, with more than a $50 \%$ reduction in medication. ${ }^{8}$ The safety data from these initial 265 patients are encouraging. There have been no incidents of erosion of the device or endophthalmitis. Nor are there reports of hypotony maculopathy or choroidal effusion. Suprachoroidal hemorrhages have not occurred, and postoperative hyphema have been infrequent, small, and brief-typically lasting less than 48 hours. Finally, the acute IOP spikes that often accompany spontaneous closure of a cyclodialysis cleft have not occurred.7,8

The current FDA study is the COMPASS clinical trial (compassclinicaltrial.com). This randomized, controlled study is twice the size of any previous microstent trials, with a total of 505 patients. Subjects are randomized either to undergo implantation of the CyPass combined with phacoemulsification or to have phacoemulsification alone. The study has a robust design, with diurnal IOP measurements at baseline, 1 year, and 2 years, all with medication washouts. The investigation will be fully enrolled in the next several months, again with follow-up for 2 subsequent years. 


\section{CONCLUSION}

Supraciliary stenting with the CyPass Micro-Stent offers a number of potential advantages over canal surgeries and trabecular stents. Because the suprachoroidal space is continuous and larger than Schlemm canal, it may have a significantly higher absorptive capacity for outflow. Moreover, because there is a greater area for stenting in the suprachoroidal space, large devices can be developed. The CyPass has almost three times the internal diameter of a trabecular microstent and six times the length, allowing additional fenestrations that may further enhance outflow. Furthermore, the placement of a suprachoroidal stent at its intended site of implantation is straightforward, is easily confirmed at the time of surgery, and can be imaged postoperatively with ultrasound biomicroscopy and optical coherence tomography (Figure 3). ${ }^{\text {? }}$

The CyPass is minimally invasive, ab interno, conjunctivasparing, nonperforating, blebless glaucoma surgery. The device creates a controlled cyclodialysis cleft that lowers IOP by filtering aqueous from the anterior chamber to the suprachoroidal space. The reported outcomes data so far are encouraging in terms of a successful and sustained reduction in IOP, and the implant appears to be well tolerated by the eye. Moreover, the problems of hypotony and bleeding-so common with cyclodialysis clefts-have not occurred with the CyPass. In this author's experience, the one-step implantation technique is straightforward and easily adopted. Nonetheless, the insertion technique is undergoing further refinement for yet simpler implantation, without the need for surgical gonioscopy. ${ }^{10}$

William J. Flynn, MD, is a glaucoma specialist in private practice in San Antonio, and he is a clinical professor of ophthalmology at The University of Texas Health Science Center at San Antonio. He is a clinical investigator for Transcend Medical in the COMPASS Clinical Trial. Dr. Flynn may be reached at (210)340-1212; flynn@rreyes.net.

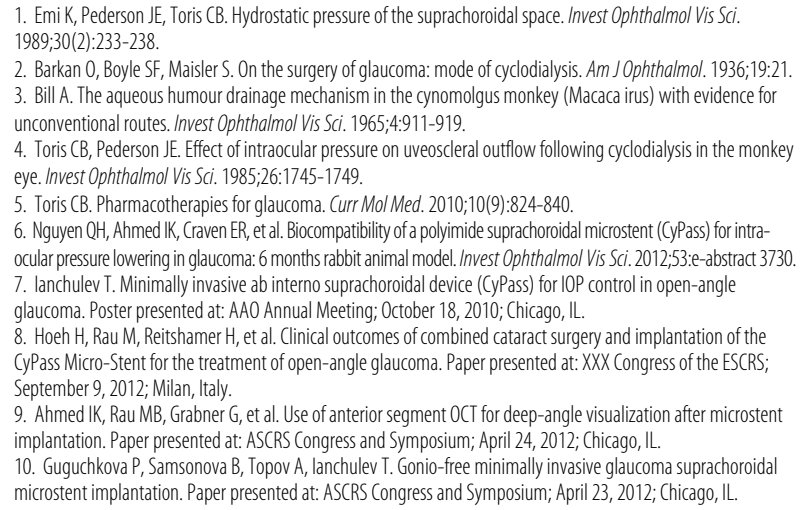

\section{The Hydrus Microstent}

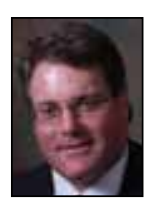

\section{By Robert Marquis MD, PhD}

The obstruction of aqueous outflow may cause ocular hypertension and glaucoma. Traditional ab externo glaucoma surgeries carry significant risks, including hypotony, choroidal effusion and hemorrhage, and bleb- or shuntrelated infections, sometimes arising years after the procedure. The clear necessity for safer yet effective surgical intervention has spawned the new class of microinvasive glaucoma surgery, and the initial results of the Hydrus Microstent (Ivantis, Inc.) in studies outside the United States have been encouraging.

\section{DESIGN}

Comparable in size to an eyelash, the Hydrus is made from nitinol, a nickel-titanium alloy. Nitinol medical devices have been implanted in various anatomical locations and have demonstrated an excellent safety and biocompatibility profile. Initial studies of the Hydrus show similar safety in the eye, as discussed later. Figure 1 shows the device's dimensions and curvature, which takes the same arc as Schlemm canal.

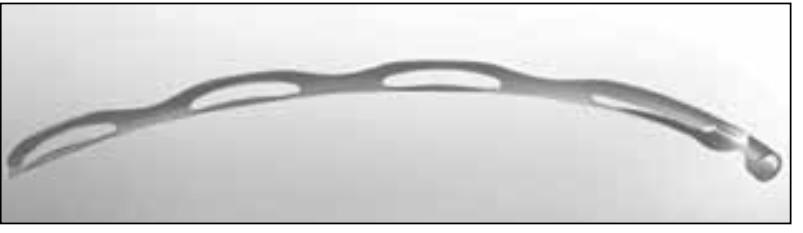

Figure 1. The Hydrus Microstent is designed to dilate 3 clock hours of Schlemm canal. The inlet at the right is positioned in the anterior chamber to facilitate aqueous flow across the trabecular meshwork and through Schlemm canal into the collector channels.

\section{PROCEDURE}

The Hydrus is loaded inside a handheld injector. At the time of surgery, the ophthalmologist observes the nasal iridocorneal angle under direct gonioscopy. The injector's tip is advanced through a temporal corneal wound, then engaged through the trabecular meshwork and delivered into Schlemm canal by the surgeon's advancing the roller wheel mechanism on the injector with his or her index finger (Figure 2).

The procedure uses topical anesthesia and is currently under investigation in the United States in patients with primary open-angle glaucoma (grade 3 or 4 ) who are 


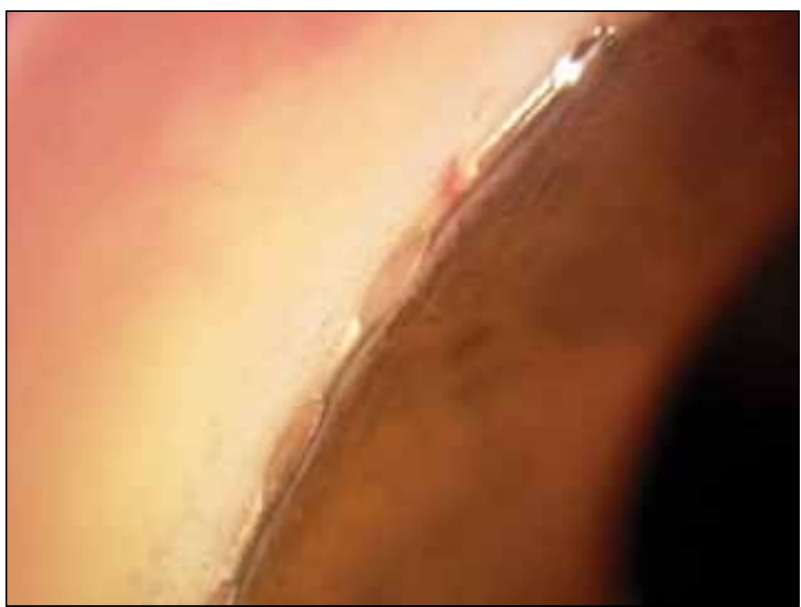

Figure 2. The device $\mathbf{3 0}$ days after its implantation into Schlemm canal. The inlet is at the top right of the image.

undergoing phacoemulsification surgery. In many cases, the cataract wound may be reused for implantation of the Hydrus, obviating the need for an additional incision. Surgical time is brief compared with that of traditional ab externo procedures.

Outside the United States, the Hydrus is being evaluated in several studies, including comparative effectiveness research against an alternative microinvasive glaucoma surgical approach, in both phakic patients and those undergoing a combined glaucoma-cataract procedure.

\section{RESULTS}

\section{Efficacy and Safety}

More than 500 Hydrus Microstents have been implanted by over 20 surgeons in 10 countries outside the United States during the past 3 years. Samuelson and colleagues reported the 6-month results of a six-center trial of

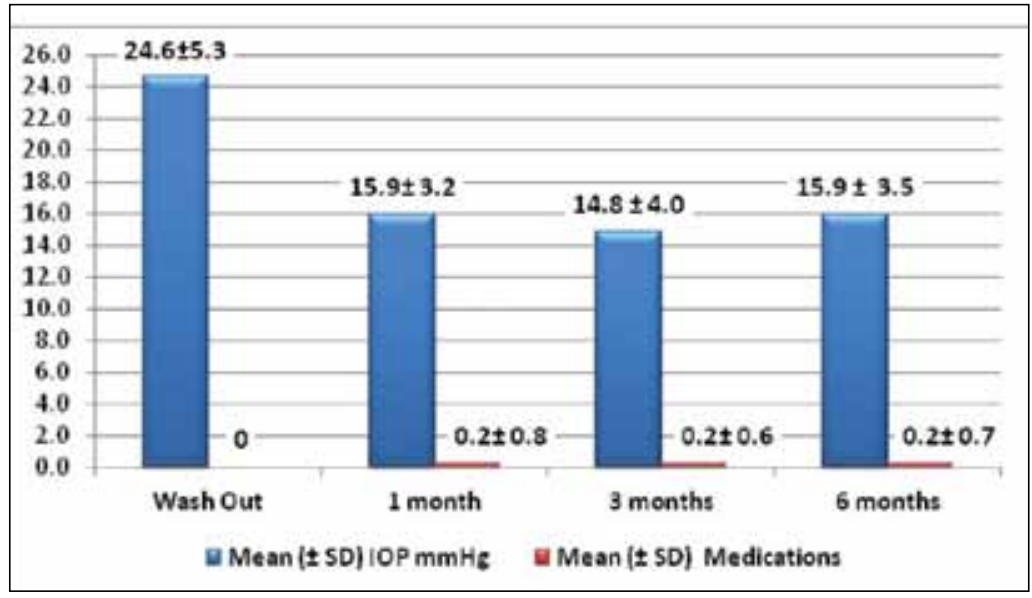

Figure 3. In the Hydrus I Study, the implantation of the device in conjunction with cataract surgery significantly reduced IOP. ${ }^{1}$
29 patients who underwent combined phacoemulsification and Hydrus implantation. ${ }^{1}$ The average preoperative IOP was $24.6 \pm 5.3 \mathrm{~mm} \mathrm{Hg}$ after washout from an average of 2.2 topical medications. The 1-, 3-, and 6-month data showed significant decreases in IOP, averaging $8.7 \mathrm{~mm} \mathrm{Hg}$ at 6 months (Figure 3). No significant complications were observed during surgery or during postoperative follow-up visits. Remarkably, $79 \%$ of patients remained free of medication with an IOP of $18 \mathrm{~mm} \mathrm{Hg}$ or less at 6 months.

In another report, Samuelson and colleagues discussed the 6-month results for an additional 40 patients who received the Hydrus as an isolated procedure (not combined with cataract extraction). ${ }^{2}$ The average preoperative IOP was $21.6 \pm 4.4 \mathrm{~mm} \mathrm{Hg}$ on an average of $1.7 \pm 1.4$ medications. The 6-month follow-up data showed a significant reduction in IOP to $16.9 \pm 3.8 \mathrm{~mm} \mathrm{Hg}$, and the number of medications needed was also reduced significantly to 0.6 (from 1.7 preoperatively). Minor complications, including transient hyphema that resolved in less than a week, were reported, but no significant complications arose.

Although both trials were limited by sample size and time of follow-up thus far, the results are encouraging and point to the need for additional studies.

\section{Current US Investigational Trial}

Presently, a 2-year prospective, randomized, controlled trial is underway in the United States to determine the safety and efficacy of the Hydrus implanted in conjunction with phacoemulsification. The control group undergoes cataract surgery alone. This study design is important to determine the effectiveness of the microstent, because it has been established that cataract surgery alone may lower IOP significantly. This trial should eventually enroll more than 500 patients at more than 30 sites.

The primary endpoint will be a reduction in IOP of at least $20 \%$ after medication washout, with results measured at the 2-year postoperative time point. The secondary endpoint will be the difference in IOP reduction between the Hydrus and control groups. Safety outcomes will include vision loss, changes found during a slit-lamp examination, endothelial cell loss, and any other complications or adverse events.

\section{CONCLUSION}

Although topical medications have improved with regard to dosing frequency and side effect profile during the past 4 decades, surgical innovations have lagged. Blebless procedures are of great interest, but an ab externo 
approach (as used in canaloplasty) necessarily compromises a portion of conjunctiva that may be needed for future surgery, whereas an ab interno approach preserves the conjunctiva. The Hydrus Microstent appears to offer some of the same benefits as canaloplasty: dilation of Schlemm canal, improved outflow through the collector channels, and decreased resistance to flow across the trabecular meshwork. The additional advantages of reduced surgical time, minimal postoperative complications, and efficient implantation hold great potential.
Robert Marquis, MD, PhD, is a glaucoma specialist at Texan Eye Glaucoma Service in Austin, Texas. He is an investigator for Glaukos Corporation and Ivantis, Inc. Dr. Marquis may be reached at (512) 327-7000; rmarquis@swbell.net.

1. Samuelson TW, Lorenz K, Pfeiffer N. Six month results from a prospective, multicenter study of a nickel-titanium Schlemm's canal scaffold for IOP reduction after cataract surgery in open angle glaucoma. Poster presented at: American Glaucoma Society 22nd Annual Meeting; March 1, 2012; New York, NY.

2. Samuelson TW, Pfeiffer N, Lorenz K. Six month results from a prospective, multicenter study of a nickel-titanium Schlemm's canal scaffold for IOP reduction in open angle glaucoma. Poster presented at: American Glaucoma Society 22nd Annual Meeting; March 1, 2012; New York, NY

\section{iStent Inject}

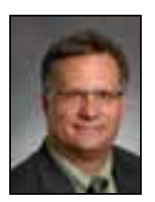

\section{By E. Randy Craven, MD}

With the FDA's clearance of a trabecular microbypass stent, the surgical options for glaucoma will continue to expand as better technology is developed. Based on the 2-year data from the first-generation iStent Trabecular Micro-Bypass Stent (Glaukos Corporation), significantly more eyes in the stent group had an IOP of $21 \mathrm{~mm} \mathrm{Hg}$ or lower without ocular hypotensive medications than in the control (phacoemulsification alone) group $(P<.036)$. In addition, the IOP with one device was $17 \mathrm{~mm} \mathrm{Hg}$ at year 1 and remained stable at $17.1 \mathrm{~mm} \mathrm{Hg}$ at year 2. In the control group, the IOP drifted upward $0.8 \mathrm{~mm} \mathrm{Hg}$ during year 1 to reach $17.8 \mathrm{~mm}$ $\mathrm{Hg}$ at year 2. These results indicate that the IOP reduction achieved with the iStent may be sustained. More patients were free of medication in the combined than the control group (61\% vs $50 \% ; P=.36$.). ${ }^{1}$ This finding indicates that patients who wish to decrease their dependence on topical IOP-lowering medication after cataract surgery may want to consider an iStent. Of course, surgeons who were not a part of the device's FDA studies are just now starting to evaluate the iStent and how it works in their hands versus in a clinical trial. One of the problems that doctors have struggled with somewhat is the bidirectional movement required for positioning of the stent in Schlemm canal. First, the surgeon must use a medial-lateral movement to get the device across the anterior chamber, and then, an inferior motion is required to position the stent in the canal. A single motion across the anterior chamber in a direction toward the trabecular meshwork would be an easier option, because only medial-lateral movement would be required.

Several years ago, lke Ahmed, MD, reported on the IOPlowering effect from the placement of more than one iStent. He found that implanting two or three stents produced a lower mean IOP than the placement of a single stent. ${ }^{2}$ Glaukos Corporation began a trial evaluating an injectable trabecular microbypass stent with two devices loaded in the

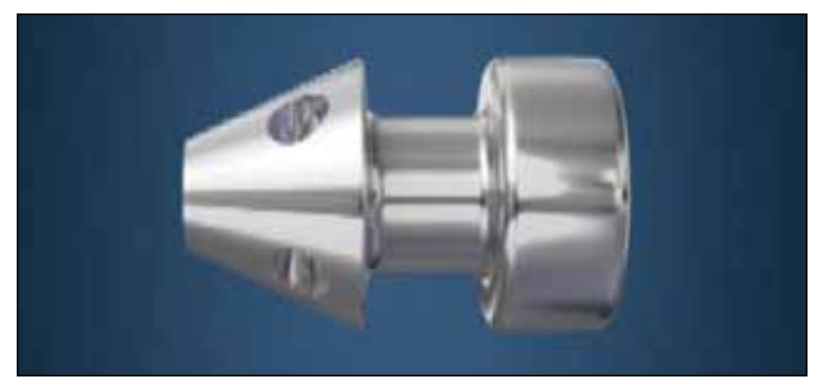

Figure 1. The flanged end penetrates the trabecular meshwork and stops with the thicker, flat end in the anterior chamber.

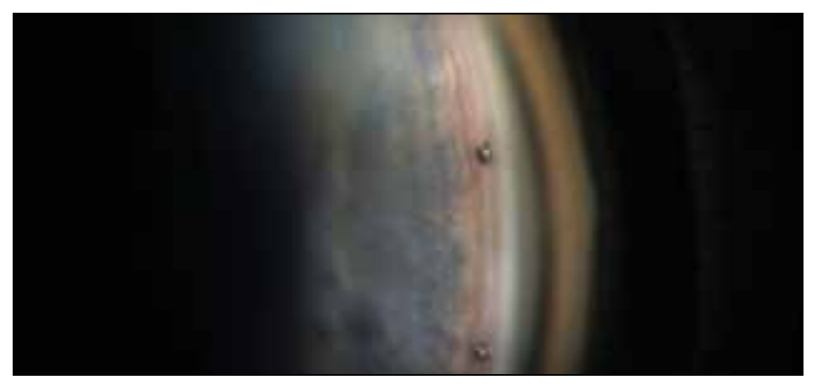

Figure 2. Two stents positioned with 2 clock hours of separation between them lowered the IOP in this eye to $12 \mathrm{~mm} \mathrm{Hg}$ on the first postoperative day from a preoperative washout level of $25 \mathrm{~mm} \mathrm{Hg}$.

same inserter (GTS 400 or the iStent Inject). This research is now a prospective, randomized, controlled, pivotal US investigational device exemption trial. The idea is to allow the surgeon to access two areas of the canal of Schlemm and to bypass the trabecular meshwork with two implants on the same inserter, using just a medial-lateral motion to get the devices into the meshwork.

\section{DESIGN}

The iStent Inject is a single-piece, heparin-coated titanium stent with a length of $360 \mu \mathrm{m}$ and a maximum width of $230 \mu \mathrm{m}$. It has four outflow orifices that divert aqueous into 
the canal. The device can be implanted in the right or the left eye (unlike the istent recently approved by the FDA that comes in right or left eye models). An outside area of reduced diameter, midway along the device, is designed to promote retention within the trabecular meshwork (Figure 1).

\section{PROCEDURE}

The sterile, single-use injector system is preloaded with two stents and designed to deliver them into Schlemm canal. The surgeon traverses the anterior chamber with the injector. Its tip has a sharpened, stainless steel trocar and a stainless steel inser-

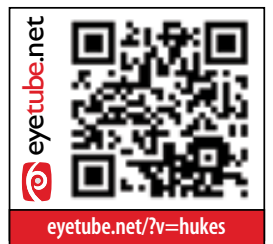
tion tube with an insertion sleeve. The stents are aligned on the trocar and are within the insertion tube, which is contained within the insertion sleeve. The injector's housing features an insertion sleeve retraction button and a stent release button. After the trocar penetrates the trabecular meshwork, the surgeon injects a stent by pushing the stent release trigger on the inserter system. After implantation, there are several clock hours of distance between the two stents (Figure 2). The idea behind using two stents is that they will get into the canal of Schlemm and, through closer proximity, tap more collector channels. This may decrease the IOP further than a single iStent.

\section{RESULTS}

Two-year results for the first 121 patients who had a medicated IOP of $24 \mathrm{~mm} \mathrm{Hg}$ or less before undergoing phacoemulsification combined with the implantation of the iStent Inject were released this year. At the time of analysis, 25 subjects who had received stents and 17 control subjects had completed 2 years of follow-up. Of those in the stent treatment group, $68 \%$ had an unmedicated IOP of $18 \mathrm{~mm} \mathrm{Hg}$ or less versus $24 \%$ of the control group. ${ }^{3}$ These results are encouraging, and the technology appears to represent an advance in the options for treating early to moderate open-angle glaucoma.

E. Randy Craven, MD, is an associate clinical professor at Rocky Vista University in Parker, Colorado. Dr. Craven is a consultant to Ivantis Inc. and Transcend Medical and a stockholder in Solx, Inc. He has received research support from Glaukos Corporation, Ivantis Inc., and Transcend Medical. Dr. Craven may be reached at (303) 748-5102; ercraven@yahoo.com.

1. Craven ER, Katz LJ, Wells JM, Giamporcaro JE. Cataract surgery with trabecular micro-bypass stent implantation in patients with mild-to-moderate open-angle glaucoma and cataract: two-year follow-up. J Cataract Refract Surg. 2012;38:1339-1345

2. Belovay GW, Ahmed II. Using multiple trabecular micro-bypass stents in cataract patients to treat primary openangle glaucoma. Paper presented at: The ASCRS Symposium and Congress; April 9-14, 2010; Boston, MA. 3. Bacharach J. Results from two years postoperative from prospective, randomized studies of second generation stents and cataract surgery in mild-moderate open-angle glaucoma. Poster presented at: American Glaucoma Society 22nd Annual Meeting; March 1, 2012; New York, NY

\section{EXCIMER LASER TRABECULOSTOMY}

By Michael S. Berlin, MD, MS; Marc TötebergHarms, MD; Lea Kleineberg, MD; Richard P. Stodtmeister, MD; and Ulrich F. Giers, MD

The primary cause of elevated IOP in most cases of openangle glaucoma (OAG) is increased outflow resistance in the juxtacanalicular trabecular meshwork (TM) and inner wall of the canal of Schlemm. ${ }^{1}$ The goal of performing laser surgery at the anterior chamber angle is to re-establish a physiological aqueous outflow pathway. Experiments with photothermal and photodisruptive lasers (ruby, Nd:YAG, and argon) were compromised by scarring through inflammatory and healing responses. ${ }^{2-11}$ Excimer laser trabeculostomy (in Europe, "trabeculotomy"; ELT) uses a nonthermal, short-pulsed, 308-nm, xenon-chloride excimer laser to ablate the TM. The pores created have sharp edges, thus avoiding scar formation. ${ }^{12-15}$

\section{PROCEDURE}

ELT can be performed as a standalone procedure or in combination with cataract surgery (phacoemulsification plus ELT). Local anesthesia is adequate. Visualization of the
TM is achieved by gonioscopy or via an endoscope. A fiber optic probe enters the anterior chamber through a paracentesis, and with the probe in direct contact with the TM, the pulsed laser energy is applied. ${ }^{16}$ In the current protocol, five to 10 pores are created over $90^{\circ}$ of the TM (Figure). Slight retrograde bleeding from the canal of Schlemm is not considered a complication but indicates patency of the created pores. The decrease in IOP is usually present from the first day after the procedure and remains stable over time. The ELT procedure takes less than 5 minutes. ${ }^{17}$

\section{RESULTS}

The first human clinical trial of ELT was performed in Germany in 1997. In 22 eyes with OAG, the median reduction in IOP was $7 \mathrm{~mm} \mathrm{Hg} .{ }^{18}$ There was only minimal trauma to the eye. Of particular note, there is no conjunctival manipulation to compromise later filtering surgery.

Since 1997, numerous clinical studies have demonstrated ELT's ability safely to achieve a long-term reduction in IOP. A retrospective study assessed 70 eyes after ELT and 60 eyes after combined phacoemulsification plus ELT that had a diagnosis of primary OAG or ocular hypertension. 


\section{COVER STORY}

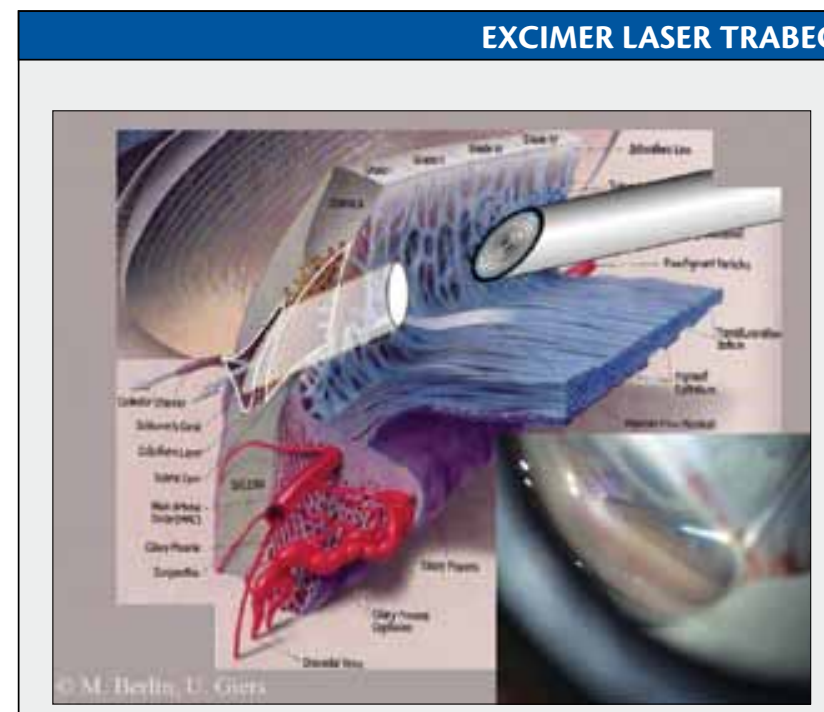

Figure. Schematic and gonioscopic view (inset) of the anterior chamber angle with a $200-\mu \mathrm{m}$ fiber-optic probe introduced through a paracentesis and in direct contact with the TM. The ablation zone of the laser from the TM to the canal of Schlemm is the shaded white area, which spares the outer wall of the canal. With a nonthermal 308-nm XeCl excimer laser, the TM, juxtacanalicular TM, and inner wall of the canal of Schlemm are ablated to create five to 10 pores.

The study had a follow-up period of up to 26 months. The investigators reported a reduction of IOP in the ELT group from $24.1 \pm 0.7 \mathrm{~mm} \mathrm{Hg}$ to $18.8 \pm 0.8 \mathrm{~mm} \mathrm{Hg}$ after 1 year and to $16.8 \pm 1 \mathrm{~mm} \mathrm{Hg}$ after 2 years (-30.3\%), while the number of antiglaucoma drugs decreased slightly (1.9 \pm 0.1 dropping to $1.5 \pm 0.3$ after 2 years). In the combined group, the IOP decreased from $22.4 \pm 0.6 \mathrm{~mm} \mathrm{Hg}$ to $16.4 \pm 0.4 \mathrm{~mm} \mathrm{Hg}$ at 1 year and $12.8 \pm 1.5 \mathrm{~mm} \mathrm{Hg}$ after 2 years (-42.9\%), while the number of antiglaucoma drugs remained almost constant (from $1.1 \pm 0.2$ to $1.2 \pm 0.2$ after 2 years). ${ }^{17}$

A recently published study exclusively analyzed combined phacoemulsification plus ELT $(n=24)$ and confirmed an immediate pressure-lowering effect. This study also confirmed a significant decrease in medication after a follow-up period of 1 year $(25.5 \pm 2.9 \mathrm{~mm} \mathrm{Hg}$ dropping to $16.5 \pm 5 \mathrm{~mm}$ $\mathrm{Hg}$, $-35.3 \%$; antiglaucoma drugs, $2.3 \pm 1.3$ dropping to $1.5 \pm 1.4$, $-34.8 \%)^{19}$

\section{CONCLUSION}

ELT is a promising, minimally invasive treatment for lowering IOP in eyes with OAG. It has been approved for use in the European Union and Switzerland for more than a decade. Currently, clinical studies and FDA approval are pending in the United States and Canada.
Michael S. Berlin, MD, MS, the director of the Glaucoma Institute of Beverly Hills, is also a professor of clinical ophthalmology at the Jules Stein Eye Institute, University of California, Los Angeles. As the inventor of ELT, he holds patents for the

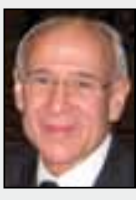
procedure in the United States and currently has patents issued and pending for next-generation ELT improvements in the United States, Canada, and the European Union. Dr.Berlin may be reached at (310) 855-1112; berlin@ucla.edu.

Ulrich F. Giers, MD, is director of the Eye Hospital, Detmold, Germany.

Lea Kleineberg, MD, is an ophthalmologist at the Eye Hospital, Detmold, Germany.

Richard P. Stodtmeister, MD, is a professor of ophthalmology at the University of Dresden, Germany.

Marc Töteberg-Harms, MD, is a clinical and basic research fellow with the Glaucoma Department at the Massachusetts Eye and Ear Infirmary, Harvard Medical School, Boston. Dr. Töteberg-Harms acknowledged no financial

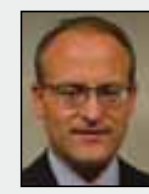
interest in the material presented herein. He received personal funding by the Swiss National Science Foundation (SNSF Project No. PBZHP3_141457). Dr. Töteberg-Harms may be reached atmarc_toeteberg@meei.harvard.edu.

1. Seiler T, Wollensak J. The resistance of the trabecular meshwork to aqueous humor outflow. Graefes Arch Clin Exp Ophthalmol. 1985;223(2):88-91.

2. Berlin MS. General aspects of laser therapy. In: Stamper RL, Lieberman MF, Drake MV, eds. BeckerShaffer's Diagnosis and Therapy of the Glaucomas. 8th ed. St. Louis, M0: Mosby; 2009:436-438.

3. Berlin MS. Laser treatment for outflow obstruction. In: Stamper RL, Lieberman MF, Drake MV, eds. Becker-Shaffer's Diagnosis and Therapy of the Glaucomas. 8th ed. St. Louis, MO: Mosby; 2009:447-455. 4. Bonney $\mathrm{CH}$, Gaasterland DE, Rodrigues MM, et al. Short-term effects of Q-switched ruby laser on monkey anterior chamber angle. Invest Ophthalmol Vis Sci. 1982;22(3):310-318.

5. Carenini BB, Nuzzi R. Laser treatment of glaucoma. Curr Opin Ophthalmol. 1992;3(2):178-189. 6. Epstein DL, Melamed S, Puliafto CA, Steinert RF. Neodymium: YAG laser trabeculopuncture in open-angle glaucoma. Ophthalmology. 1985;92(7):931-937.

7. Gaasterland DE, Bonney CH 3rd, Rodrigues MM, Kuwabara T. Long-term effects of Q-switched ruby laser on monkey anterior chamber angle. Invest Ophthalmol Vis Sci. 1985;26(2):129-135.

8. Krasnov MM. Laseropuncture of anterior chamber angle in glaucoma. Am J Ophthalmol. 1973;75(4):674-678. 9. Krasnov MM. Q-switched laser goniopuncture. Arch Ophthalmol. 1974;92(1):37-41.

10. Melamed S, Latina MA, Epstein DL. Neodymium:YAG laser trabeculopuncture in juvenile open-angle glaucoma. Ophthalmology. 1987:94(2):163-170.

11. Shingleton BJ, Richter CU, Dharma SK, et al. Long-term efficacy of argon laser trabeculoplasty. A 10-year follow-up study. Ophthalmology. 1993;100(9):1324-1329.

12. Jahn R, Lierse W, Neu W, Jungbluth KH. Macroscopic and microscopic findings after excimer laser treatment of different tissue. J Clin Laser Med Surg. 1992;10(6):413-418.

13. Walker R, Specht H. Theoretical and physical aspects of excimer laser trabeculotomy (ELT) ab interno with the AIDA laser with a wave length of $308 \mathrm{~mm}$ [in German]. Biomed Tech (Berl). 2002;47(5):106-110.

14. Berlin MS, Rajacich G, Duffy M, et al. Excimer laser photoablation in glaucoma filtering surgery. Am J Ophthalmol. 1987;103(5):713-714.

15. Berlin MS, Yoo PH, Ahn RJ. The role of laser sclerostomy in glaucoma surgery. Curr Opin Ophthalmol. 1995;6(2):102-114.

16. Pache M, Wilmsmeyer S, Funk J. Laser surgery for glaucoma: excimer-laser trabeculotomy [in German]. Klin Monbl Augenheilkd. 2006;223(4):303-307.

17. Wilmsmeyer S, Philippin H, Funk J. Excimer laser trabeculotomy: a new, minimally invasive procedure for patients with glaucoma. Graefes Arch Clin Exp Ophthalmol. 2006;244(6):670-676.

18. Vogel M, Lauritzen K. Selective excimer laser ablation of the trabecular meshwork. Clinical results [in German]. Ophthalmologe. 1997;94(9):665-667.

19. Toteberg-Harms M, Ciechanowski PP, Hirn C, Funk J. One-year results after combined cataract surgery and excimer laser trabeculotomy for elevated intraocular pressure [in German]. Ophthalmologe. 2011;108(8):733-738 


\section{iStent Supra}

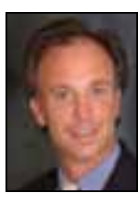

By Jason Bacharach, MD

It is an exciting but challenging moment for physicians who treat patients with glaucoma. Surgeons desire less invasive techniques with quicker rehabilitation times, higher efficiency in the $\mathrm{OR}$, and the ability to combine glaucoma procedures with cataract surgery. Microinvasive glaucoma surgery is characterized as a microincisional technique that involves no destruction of tissue, no corneal flaps, and no disruption of the conjunctiva. On the heels of the FDA's approval of the iStent Trabecular Micro-Bypass Stent (model G-1), Glaukos Corporation has initiated clinical trials to develop a suprachoroidal device (the iStent Supra). The goal is to allow surgeons to lower IOP by enhancing uveoscleral or conventional outflow, both via an ab interno approach.

The iStent Supra is one of a few new technologies that attempt to harness a potentially vacuum-like effect of the suprachoroidal space by shunting aqueous to the area. Once in the suprachoroidal space, the aqueous exits either via a transscleral route or by choroidal absorption. Other technologies under investigation for use in the suprachoroidal space are the Solx Gold Shunt

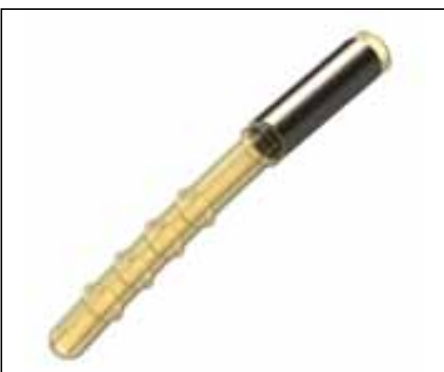

Figure 1. The iStent Supra.
(Solx, Inc.) and the CyPass Micro-Stent (Transcend Medical). I have been a clinical investigator of and have experience placing both the Solx Gold Shunt (via an ab externo approach) and the iStent Supra (via an ab interno approach). (For more on the Solx Gold Shunt, see the sidebar by Marlene R. Moster, MD, on this page.)

\section{DESIGN}

The iStent Supra is designed to create a patent lumen between the anterior chamber and the suprachoroidal space. It is made of poyethersulfone and has a colored titanium sleeve. The device also has a proprietary heparin coating (Duraflo) and is biocompatible. It is nonpyrogenic, nonferrous (not magnetic), and terminally sterilized (irradiation). The stent is slightly curved to match the suprachoroidal space. It features retention rings to provide stability at the site of implantation (Figure 1).

\section{THE SOLX GOLD SHUNT}

\section{By Marlene R. Moster, MD}

Successfully treating patients with refractory glaucoma remains an unmet surgical challenge. The Solx Gold Shunt (Solx, Inc.) has the potential to address this population without the formation of a bleb. This minimally invasive device is made of pure biocompatible gold and is placed in the suprachoroidal space via an elegant surgical procedure (Figure). The device is approved for use in Canada and Europe, and enrollment in its phase 3 US investigational device exemption trial is nearing completion. Although it is too early to report on the study's results, the concept behind the device and previously reported data indicate that this shunt may represent an attractive future option for glaucoma specialists.

Marlene R. Moster, MD, is a professor of ophthalmology at the Thomas Jefferson School of Medicine and is an attending surgeon at Wills Eye Institute, both in Philadelphia. She acknowledged no financial interest in the product or company mentioned herein. Dr. Moster may be reached at (215) 928-3342; marlenemoster@aol.com.

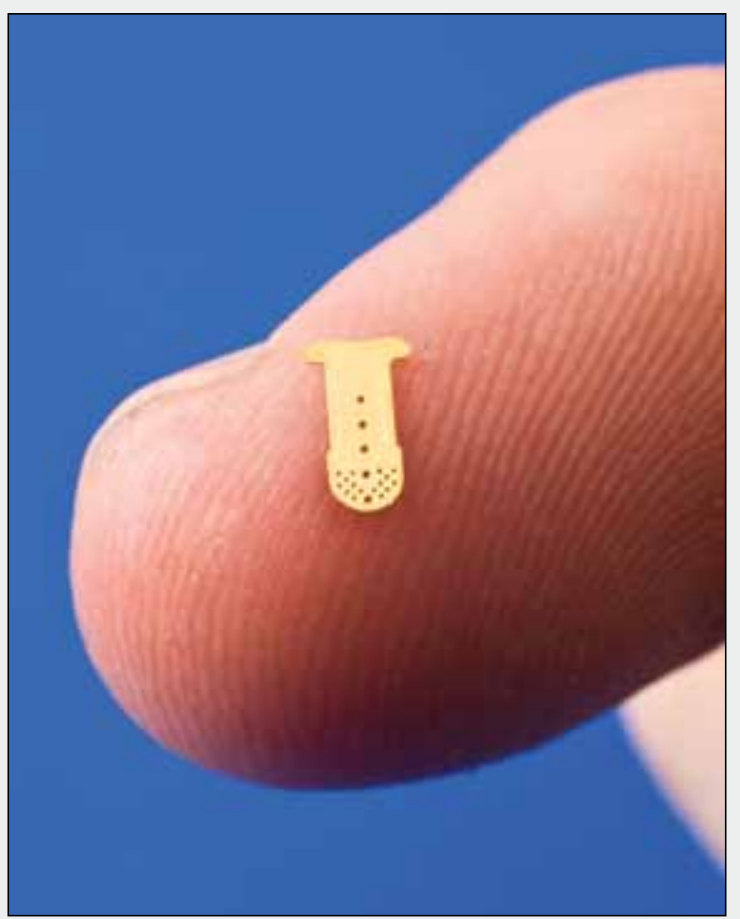

Figure. The Solx Gold Shunt. 


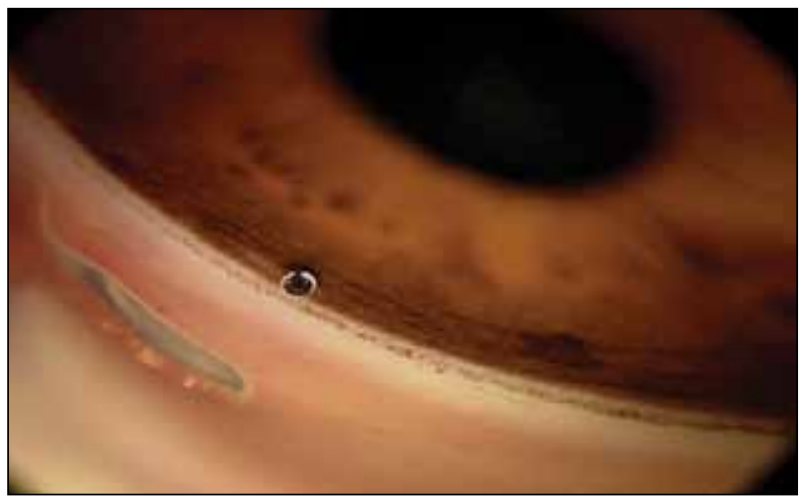

Figure 2. The iStent Supra in the suprachoroidal space.

\section{PROCEDURE}

The iStent Supra can be implanted in combination with cataract surgery or as a freestanding technique. The keys to successfully implanting the suprachoroidal device are clear visibility of the angle structures and a knowledge of the angle landmarks. The surgeon views the angle through a goniolens placed on the cornea. My tips for optimizing visibility are tilting the patient's head away from the surgeon and zooming up the microscope's power $(10 x-12 x)$. The stent is implanted right below the scleral spur. Next, the trocar/stent is advanced into the suprachoroidal space until approximately $0.5 \mathrm{~mm}$ of the sleeve is left in the anterior chamber. Then, the surgeon engages a trigger mechanism on the inserter to release the stent (Figure 2).

\section{RESULTS}

Earlier this year, Eric Donnenfeld, MD, presented the results of a prospective, single-arm, open-label study of 40 subjects (current enrollment, 37 eyes). ${ }^{1}$ The subjects had moderate to advanced glaucoma (cup-to-disc ratio $\leq 0.9$ ) and no history of surgical intervention for glaucoma. Their preoperative IOP was 18 to $30 \mathrm{~mm} \mathrm{Hg}$ on two medications (mean of currently enrolled subjects = $20.7 \pm 2.5 \mathrm{~mm} \mathrm{Hg}$ ). Baseline IOP after washout was 22 to $38 \mathrm{~mm} \mathrm{Hg}$ (mean of currently enrolled subjects $=24.7$ $\pm 1.9 \mathrm{~mm} \mathrm{Hg}$ ). The treatment included one iStent Supra and one postoperative medication (travoprost). The study's protocol includes 2 years of follow-up.

Dr. Donnenfeld reported the status of 25 subjects through 1 year. The mean decrease in IOP at this time point was $43 \%$, with a reduction of one medication. Ninety-six percent of patients met the primary efficacy endpoint of at least a $20 \%$ reduction in IOP. All eyes met the secondary endpoint of an IOP that was less

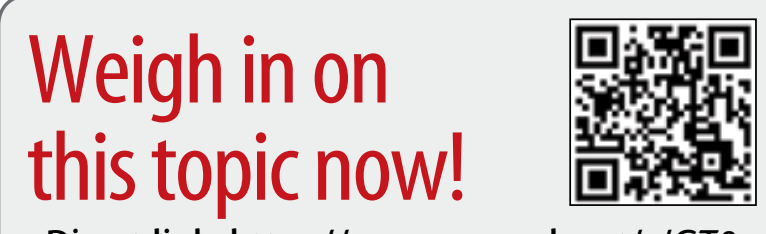

Direct link: https://www.research.net/s/GT8

In which of the following technologies not available in the United States are you currently the most interested?

$\square$ AqueSys Implant

$\square$ CyPass Micro-Stent

$\square$ Excimer Laser Trabeculostomy

$\square$ Hydrus Microstent

$\square$ iStent Inject

$\square$ iStent Supra

$\square$ Solx Gold Shunt

than or equal to $18 \mathrm{~mm} \mathrm{Hg}$ (the IOP was $\leq 15 \mathrm{~mm} \mathrm{Hg}$ in $80 \%$ of subjects). There were no untoward adverse events intra- or postoperatively. Dr. Donnenfeld and his fellow investigators concluded that implanting the iStent Supra as a sole procedure is feasible, safe, and capable of significantly reducing IOP and the need for topical medical therapy in patients with open-angle glaucoma that was previously uncontrolled on two agents. The study is ongoing, and 2-year data are being collected.

\section{CONCLUSION}

The flipside to the excellent safety profile of microinvasive glaucoma surgery is that, in general, it lowers IOP less than more invasive procedures (ie, trabeculectomy and tube shunt surgery). An ab interno suprachoroidal shunt such as the iStent Supra may combine the best of these properties by achieving a lower IOP than $a b$ interno stenting of Schlemm canal while maintaining conjunctival integrity.

Jason Bacharach, MD, is the director of research at North Bay Eye Associates, Inc., in Sonoma, California, vice-chair of the Glaucoma Department at California Pacific Medical Center in San Francisco, and chair of the American Academy of Ophthalmology's Practicing Ophthalmologists Curriculum Glaucoma Panel. He is an investigator for and consultant to Glaukos Corporation. Dr. Bacharach may be reached at (707) 762-6622; jb@northbayeye.com.

1. Donnenfeld ED. Open-angle glaucoma treated with implantation of suprachoroidal stent and topical travoprost. Paper presented at: ASCRS Symposium and Congress; April 20-24, 2012; Chicago, IL. 